\title{
Zehn Jahre nach den Arabischen Umbrüchen: Kritische Reflektionen zu Medien und Transformation in Ägypten und Tunesien
}

\author{
Hanan Badr
}

Keywords: Arabische Umbrüche, Soziale Medien, Demokratisierung, Transformation

\section{Abstract}

Der Beitrag plädiert für ein kontextsensibles Verständnis von Medien und Transformation im Rahmen der Arabischen Umbrüche, die vor zehn Jahren die arabische Region und die Welt erschütterten. Durch die Entwicklung und Anwendung des Begriffs counter-issues, die sozioökonomische und politische Missstände thematisierten und Menschen mobilisierten, geht der Artikel über die Kontroverse der Rolle von Medien oder Politik beim Auslösen der Transformationsprozesse hinaus. Der Beitrag fasst abschließend die theoretischen Implikationen für die Erforschung von Transformation und Medien in nicht-westlichen Kontexten, mit besonderem Blick auf Ägypten und Tunesien, zusammen. Transformationsprozesse können nur in ihren Kontexten verstanden werden. Die Kommunikationswissenschaft wird von einer Diversifizierung der epistemischen Perspektiven profitieren.

Hanan Badr: Zehn Jahre nach den Arabischen Umbrüchen: Kritische Reflektionen zu Medien und Transformation in Ägypten und Tunesien. In: Nils S. Borchers, Selma Güney, Uwe Krüger und Kerem Schamberger (Hrsg.): Transformation der Medien - Medien der Transformation. Verhandlungen des Netzwerks Kritische Kommunikationswissenschaft. Frankfurt am Main: Westend 2021. DOI: https://doi.org/10.53291/MVLJ2171.

Prof. Dr. Hanan Badr I Gulf University for Science and Technology (GUST), Kuwait I badr.h@gust.edu.kw 
Die Arabischen Umbrüche ${ }^{1}$ in den Jahren 2010 und 2011 haben eine heftige Debatte über die Rolle der Medien in der politischen Transformation ausgelöst. Obwohl der euphorische Moment der Massenmobilisierung vor zehn Jahren vergangen ist, hat diese Diskussion ihre Relevanz nicht verloren. Der zeitliche Abstand verhilft zur notwendigen Distanz, um kritisch über Transformationsprozesse und ihre politischen und medialen Rahmenbedingungen zu reflektieren.

Heute spricht die Washington Post vom »tragischen Erbe« der arabischen Umbrüche: Die erste Welle der Massenproteste in Tunesien, Ägypten, Libyen, dem Jemen und Syrien zog gravierende Konsequenzen wie Destabilisierung, Re-Autokratisierung und zahlreiche Bürgerkriege nach sich; die sozioökonomische und politische Ungleichheit blieb bestehen. Die zweite Protestwelle, die 2018 und 2019 im Sudan, Libanon, Irak und Algerien ausbrach, lancierte erneut die Debatte über Medien und politische Transformation. Beide Ereignisse sind Zeichen für die erdrückende strukturelle Ungleichheit und mangelnde Selbstbestimmung, gegen welche die Menschen trotz Repression und Polarisierung immer noch protestieren.

Die arabischen Umbrüche führten zu einem kurzlebigen Paradigmenwechsel in der Forschung über die arabische Region, der anstelle von Stagnation die Dynamik in den Mittelpunkt rückte (Harders 2018). Der Beginn der politischen Transformationsphase in Nordafrika geschah umbruchartig und nicht durch kontrollierte Reformen. So ersetzte dies für eine kurze Zeit die Forschung zum »enduring authoritarianism《 (Bellin 2012), bevor die regionalen Ereignisse den wissenschaftlichen Blick wieder zunehmend auf Re-Autokratisierungsprozesse richteten (Kneuer und Demmelhuber 2016). In beiden Fällen, unabhängig davon, ob der Fokus auf Stagnation oder Dynamik liegt, sollte die Region nicht als Ausnahmefall konstruiert werden, also weder als demokratieresistent noch als revolutionsfreudig. Denn beide Perspektiven verstärken einen »arab exceptionalism « in Bezug auf Medien und Transformation und reproduzieren somit einen postkolonialen westlichen Blick (Harik 2006; Harders 2018). Ohne das Einbetten kommunikativer Phänomene in ihre regionalen Kontexte verstärkt sich das epistemische Dilemma

1 Der Artikel spricht bewusst nicht vom »Arabischen Frühling «, weil dieser Begriff essentialistisch und eine Fremdzuschreibung von der westlichen Perspektive auf die regionalen Verhältnisse darstellt (Beck 2015; Harik 2006). 
der Wissensproduktion durch den externen Blick auf die unberechenbaren Araber*innen (Said 2003).

In der Erforschung der arabischen Umbrüche treffen zwei Literaturstränge und Disziplinen aufeinander, die Interdependenzen zwischen Medien und Transformation erklären. Als thematische Schnittstelle zwischen Politikwissenschaft und Regionalforschung einerseits sowie Medien- und Kommunikationswissenschaft andererseits brach eine intensive Debatte über den Zusammenhang von Medien und Transformationsprozessen in Nordafrika aus. Der epistemische Standpunkt jeder Disziplin gestaltet die Perspektive auf die Medien. Politikwissenschaft und Regionalforschung betonen die Transformationsprozesse im Sinne von Demokratisierung und Regimewechsel, die Kommunikationswissenschaft dagegen untersucht die Auswirkungen der Digitalisierung auf die politischen und gesellschaftlichen Dynamiken.

Der Begriff der Transformation ist in der Kommunikationswissenschaft zentral und bezieht sich auf den strukturellen digitalen Wandel, der diverse komplexe Prozesse in Gang setzt (Strippel 2020). Das allgegenwärtige Internet und soziale Plattformen führten zu dramatischen gesellschaftlichen Veränderungen bezüglich der Art und Weise, wie wir miteinander kommunizieren, die Welt wahrnehmen und handeln. Diskursive und mediale Aushandlungsprozesse von Identität, Deutungskämpfen und Selbstdarstellung formieren sich unter neuen kommunikativen Bedingungen der Interaktion. Die digitalen Öffentlichkeiten bringen Potenziale von Kooperation, aber auch Dissonanz und Polarisierung (Pfetsch 2018).

In der Politikwissenschaft knüpfen die Untersuchungen der arabischen Umbrüche an die Demokratisierungsforschung zu politischen Umwälzungen in Südeuropa, Lateinamerika und Osteuropa an. Dabei wird Transformation als ein Prozess verstanden, bei dem sich die Transition von der Autokratie zur Demokratie in grob drei Phasen vollziehen kann (siehe O'Donnell und Schmitter 2013): 1) Zusammenbruch des alten Regimes, 2) Aushandlung neuer Grundlagen in der Übergangsphase und 3) Konsolidierung der neuen Demokratie. Die Theorie schließt einen Rückfall in autoritäre Konstellationen nicht aus und macht dies abhängig von vielen begünstigenden oder hemmenden Faktoren in den jeweiligen Kontexten (Merkel 2010). Diese Perspektive begünstigt einen euro- und angloamerikanisch-zentrierten Blick, der auf der veralteten These beruht, dass die Ausbreitung der Demokratie auf der Welt zum internationalen Frieden beiträgt, da sich demokratische Staaten nicht bekriegen würden (Maoz und Russett 1993; Kritik dazu Rosato 2003). 
Anlehnend daran, nannten Expert*innen die arabischen Umbrüche noch in der ersten euphorischen Phase die »vierte Welle der Demokratisierung « (Howard und Hussain 2013). Ähnlich wie in den westlichen Demokratien wurden den Sozialen Medien optimistische Potenziale zur Stärkung der Demokratie und Steigerung der politischen Teilhabe zugeschrieben. Auch in Nordafrika herrschte bezüglich der Proteste in den autoritären Regimes anfänglich die vereinfachte Annahme, dass Onlinemedien und soziale Plattformen der politischen Liberalisierung dienten. Aus heutiger Perspektive wissen wir, dass dies nicht der Fall war. Eine erhoffte Befreiung durch die Medien allein kam nicht zustande. Die meisten Länder, deren Regimes zusammenbrachen - mit Ausnahme Tunesiens - gerieten in abschreckende gesellschaftliche Konstellationen von Polarisierung, politischem Chaos und Bürgerkrieg oder wandelten sich zu noch repressiveren Autokratien. Selbst Tunesien hat - trotz des Ausbleibens größerer Konflikte und Blutvergießens - noch einen langen und unsicheren Weg vor sich, um die Ursachen der systemischen Ungleichheiten zu beheben.

Dieser Beitrag blickt kritisch auf die komplexen Wechselbeziehungen zwischen Mediensystemen und Transformationen im ersten Umbruchsmoment 2010/11 kurz vor dem Zusammenbruch der Regimes in Tunesien und Ägypten. Anhand empirischer Daten im Rahmen des DFG-Forschungsprojekts »Media Functions in Transition « an der Freien Universität Berlin reflektiert der Artikel über die doppelte Bedeutung der Transformation, sowohl in politischer als auch in kommunikationswissenschaftlicher Hinsicht. Methodisch basiert das DFG-Projekt auf qualitativen Inhaltsanalysen von Printmedien und Sozialen Medien im Zeitraum von 2008 bis 2011 sowie Interviews mit Journalist*innen und Aktivist*innen, um die Rolle der Medien in der Transformationsphase zu rekonstruieren. Somit überbrückt diese Untersuchung die getrennten Perspektiven und bringt sie in Dialog. Ziel ist es dabei, die Zusammenhänge von Medien und Transformationsprozessen in ihren regionalen Kontexten zu verstehen. Anlehnend an den Begriff der hybriden Mediensysteme (Chadwick 2017) rekonstruiert der Beitrag aus einer vergleichenden Perspektive die Rolle der Medien bei der Auslösung der Transformationsprozesse in Nordafrika. »Hybridität« bezieht sich dabei auf die Notwendigkeit, die Online-offline-Interaktionen innerhalb von Mediensystemen ganzheitlich zu betrachten, ohne sie binär entweder in alte oder neue Medien zu unterteilen. Der Beitrag widerspricht somit der techno-deterministischen »Facebook-Revolution«. 
Der folgende Artikel besteht aus drei Teilen: Der erste Teil diskutiert kritisch die Kontroverse, ob Medien oder Politik die Transformationsprozesse auslösten. Der zweite Teil wendet das Konzept der counter-issues anhand von empirischen Beispielen aus Tunesien und Ägypten an, um ein kontextsensibles Verständnis von Medien und Transformation zu fördern. Dabei handelt es sich um kritische Themen, die sozioökonomische und politische Missstände thematisieren, weshalb die Machthaber sie von der Öffentlichkeit fernhalten wollen (Mathes und Pfetsch 1991; Pfetsch et al. 2016; Badr 2019). Der dritte und letzte Teil fasst abschließend die theoretischen Implikationen für die Erforschung von Transformation im Zusammenhang mit Medien und besonderem Blick auf Ägypten und Tunesien zusammen.

\section{Die Kontroverse: Die Medien zuerst oder die Politik zuerst?}

Die Frage zur Rolle von Medien bei der Auslösung der Transformationsprozesse in Nordafrika hat zwei gegensätzliche Thesen vorangetrieben: Medien zuerst (»media first«; Abdulla 2011; Khamis und Vaughn 2011) versus Politik zuerst (»politics first«; Wolfsfeld et al. 2013).

\subsection{Zuerst die Medien: Medien fördern die Liberalisierung}

Gemäß dem Standpunkt »Medien zuerst« waren es primär die Medien, die den Beginn der Transformationen auslösten. Somit konzentriert er sich auf die politische Öffnung, die durch die intensive mediale Kommunikation in Tunesien und Ägypten forciert wurde. Unmittelbar nach den Ereignissen des Jahres 2011 wies die empirische Forschung aus den arabischen Ländern auf die starke Wirkung der Sozialen Medien und deren initiierende Rolle bei den politischen Transformationsprozessen hin. In öffentlichen und medialen Diskursen wird diese These als »Facebook-Revolution « bezeichnet und auch seitens der Plattformenunternehmen selbst aufgriffen: Facebook, Twitter und Co. fördern weiterhin dieses Narrativ (Richter und Badr 2018).

Drei Argumente sprachen für diese Anfangseuphorie: Das erste lautet, dass das Internet die Öffentlichkeit dynamisiert und erweitert hat. Die These über die »liberation technology« (Diamond 2010, 96) beruht auf der Prämisse, dass die zwischenmenschliche Kommunikation au- 
ßerhalb der regulierten Massenmedien zunehmend zu einem Empowerment der marginalisierten Akteur*innen führt. Somit verschaffen sich diese einen sichtbareren Platz in den Diskurssphären.

Soziale Medien ermöglichten eine dezentralisierte Kommunikation, so dass Teilöffentlichkeiten in alternativen Onlineräumen an den Kommunikationsprozessen teilnehmen konnten. Durch das Internet kamen marginalisierte Akteur*innen, Jugendliche und oppositionelle soziale Bewegungen verstärkt zu Wort und forderten politische und religiöse Autoritäten heraus (Ghonim 2012). Trotz einer starken Internetzensur, vor allem in Tunesien, konnten zivilgesellschaftliche Akteur*innen die neuen Technologien insofern nutzen, dass sie mittels Blogs und Sozialer Medien kritische Tabuthemen aufgriffen, die von etablierten Medien übersehen oder ausgeblendet wurden. Damals schienen die Onlinediskurse die normativen Bedingungen einer idealen Öffentlichkeit zu erfüllen, da sie allen Nutzer*innen zugänglich waren und gleichberechtigte Teilhabe ermöglichten (Lynch 2014; Ayish 2008). Akteur*innen umfassten Interessengruppen, Bürgerinitiativen und soziale Bewegungen, die ansonsten aufgrund politischer Ausgrenzung oder zu schwacher Ressourcen nur begrenzten Zugang zum Mediensystem gehabt hätten. Insofern standen die autoritären Regimes diesem strukturellen Wandel der Kommunikation unvorbereitet gegenüber. Das Internet war nicht im gleichen Maße wie die traditionellen Medien reguliert und überwacht, so dass es den Akteur*innen mehr Freiräume als die Offline-Welt bot.

Das zweite Argument besagt, dass kommunikative digitale Handlungen eine Form der politischen Teilhabe darstellen. Dabei dient die Kommunikation selbst als eine aktive Form der Bürgerbeteiligung, die Individuen von passiven Konsument*innen zu aktiven Produzent*innen macht. Das Internet stellte dadurch die Möglichkeit offen, die Inkongruenz zwischen der politischen Struktur, also den Institutionen, der politischen Kultur und der Summe aller Einstellungen in einer Gesellschaft, auszugleichen. Laut der Liberalisierungsthese hat das Internet den Bürger*innen ermöglicht, die Öffentlichkeit zurückzufordern und damit Pluralismus und Vielfalt in die politische Diskussionskultur einzubringen (Rinke und Röder 2011).

Das dritte Argument, das den Einfluss digitaler Plattformen auf die politische Kommunikation erklärt, ist die intermediale Agenda-SettingFunktion. Vor den Umbrüchen in den Jahren 2010 und 2011 fand ein Agenda-Transfer der counter-issues von den Sozialen Medien auf die Mainstreammedien statt (Badr 2019). Beachtet man die digitale Un- 
gleichheit in der Verteilung von Infrastruktur, Internetzugang und Endgeräten zu dem Zeitpunkt der arabischen Umbrüche vor zehn Jahren, dann scheint die intermediale Themensetzung von Online- in Offlinemedien sogar noch wichtiger. Durch diesen Mechanismus wurden Themen für breitere Teile der Öffentlichkeit sichtbarer und zugänglicher, die diese Diskurse sonst nicht wahrgenommen hätten. Journalist*innen, vor allem in privaten Medien, mussten somit auf Themeninputs reagieren, die von Teilöffentlichkeiten kamen, um ihre gesellschaftliche Relevanz und Glaubwürdigkeit nicht zu verlieren.

Die »Medien-zuerst«-Argumente fördern eine westlich-zentrierte Perspektive, welche die neuen Technologien für eine Transformation in autoritären Kontexten begrüßt (Richter und Badr 2018). Außerdem übersah dieser Forschungsstrang die »alten « Medien in einer hybriden Umgebung, die durch symbiotische Verknüpfungen zwischen digitalen und nicht-digitalen Formaten zur Transformation beitrugen.

Da sich aus den politischen Transformationsprozessen keine neuen demokratischen Strukturen ergaben, endete diese Kontroverse zwischen der Kommunikations- und Regionalwissenschaft recht bald. Die Forschung zu den Rollen neuer Medien im digitalen Zeitalter verlagerte sich schnell auf internationale Kontexte außerhalb der arabischen Region, bei der die politische Kommunikation in den erforschten Kontexten auf Populismus, Protestkommunikation und Polarisierung fokussierte. Ein Großteil der Literatur benutzte die Begrifflichkeit des »Arabischen Frühlings« nur als Namedropping oder Relevanzbegründung der eigenen empirischen Arbeit (Richter und Badr 2018). Das Forschungsinteresse der Kommunikationswissenschaft an Medien und Transformation in der arabischen Region kam, mit Ausnahme einer geringen Zahl an Forschenden, die sich inhaltlich schon immer mit dieser Region befasst hatten, zum Erliegen (zum Beispiel El-Issawi 2021, El-Nawawy und Khamis 2013, Hafez 2014). So endete auch der mögliche multidisziplinäre Dialog zwischen der Kommunikationswissenschaft und der Regionalforschung, die darauf ausgerichtet war, die Transformations- und Aushandlungsprozesse im Zusammenhang mit den arabischen Umbrüchen nachhaltig zu verstehen. Stattdessen rückten Themen auf die europäische Forschungsagenda, welche die eigenen Sicherheitsinteressen im Blick hatten und pragmatische Ziele wie die Eindämmung von Terrorismus und Migration verfolgten (Teti et al. 2020). 


\subsection{Zuerst die Politik: (Digitale) Medien schaffen allein keine Transformation}

Die Gegenthese zur Liberalisierung durch digitale Medien analysiert die Transformationsprozesse mit Blick auf politische Machtverhältnisse: Für die Anhänger*innen dieser These gilt, dass die Politik (und nicht die Medien) an erster Stelle zur Erklärung der Massenmobilisierung steht (Wolfsfeld et al. 2013). Zwei Argumente erläutern ihre Rolle in der Entstehung von Transformationsprozessen. Das erste betrachtet die neuen Medien differenzierter: Sie sind keine absoluten Mittel zur Ermächtigung und folglich besteht kein Befreiungsautomatismus. Zwar führte die Technologie zu neuen kreativen und kommunikativen Synergiekonstellationen, doch das technologische Werkzeug selbst löste keine Revolution aus. Stattdessen mobilisierten reale Missstände die Menschen. Laut der Studie »Arab Social Media« (Salem 2017) sind sowohl die Zahlen der Internetnutzung als auch die Unzufriedenheitsraten heute größer als vor 2011. Doch Zugang zur digitalen Öffentlichkeit und hohe Nutzer*innenzahlen allein führen nicht zu erneuten Protesten (Badr 2015). Zusätzlich weisen empirische Indikatoren darauf hin, dass die bloße Abschaltung des Internets in Ägypten 2011 und im Sudan 2018 der Massenmobilisierung keinen Einhalt gebieten konnte. Dies spricht für einen kontextualisierten Blick auf die Wirkung digitaler Kommunikation auf das politische Geschehen. Eine Perspektive, welche die digitale Ungleichheit berücksichtigt, wäre treffender: Mehr Kommunikation ist nicht unbedingt befreiend, da sie nicht immer Empowerment der Nutzer*innen bewirkt. Es zählt mehr, wie die Menschen die digitalen Möglichkeiten nutzen und was sie damit bewirken können (Trappel 2019; Ragnedda und Muschert 2016).

Das zweite Argument beruht auf der These des autoritären Lernens. Darunter versteht man, dass die autoritären Regimes ebenso anpassungsfähig sind wie die unterdrückten Bürger*innen. Entsprechend haben sie gelernt, wie sie mit den neuen kommunikativen Herausforderungen umgehen müssen, um die digitale Öffentlichkeit mitzugestalten und zu kolonisieren. Im Jahr 2010 zeigte Mubaraks Antwort »Lasst die Jugend Spaß am Internet haben«, als er nach den jungen FacebookNutzer*innen gefragt wurde, wie die autoritären Regimes die Möglichkeiten der digitalen Öffentlichkeit noch unterschätzten. Seitdem haben die arabischen Regierungen gelernt, diese ernster zu nehmen (Badr 2013). 
Autoritäres Lernen umfasst drei Strategien: 1) Die Machthaber entwickelten digitale Zensur- und Überwachungsstrategien. Die berüchtigte Firewall mit dem Spitznamen »Ammar 404« in Ben Alis Tunesien war noch harmlos, wenn man sie mit den heutigen Sperrtechnologien vergleicht (Raoof 2017). Seit 2018 zum Beispiel wurden der ägyptischen Öffentlichkeit durch Sperr- und Filtertechnologien das Lesen von mehr als 700 arabischen und nicht-arabischen Websites verboten. Zusätzlich sind dort seit 2017 neue Gesetze zur Bekämpfung von Terrorismus und Cyberkriminalität Methoden eines »legalen Autoritarismus« (Hamzawy 2017, 392) geworden. Das bedeutet, dass die Regierungen versuchen, den Schein eines Rechtsstaats zu erwecken, indem sie restriktive Gesetze verabschieden und anwenden, um die digitale Öffentlichkeit enger zu kontrollieren. Administrator*innen von populären Accounts der Opposition in Sozialen Medien müssen nun mit Haftstrafen rechnen. 2) Die arabischen Regimes lernten mit Diskursen über Stabilität und nationale Sicherheit, restriktive Maßnahmen vor der Öffentlichkeit zu rechtfertigen (Elswah und Howard 2020). Trotz kritischer Äußerungen des EU-Parlaments exportieren die europäischen Länder immer noch westliche Überwachungstechnologie an Autokratien. Somit fördern sie eine autoritäre Agenda, welche die nationale Sicherheit an erster Stelle setzt, und untergräbt damit zivilgesellschaftliche Initiativen und Partnerschaften in der Region, die sich eine Selbstzensur auferlegen muss. 3) Schließlich bedient sich das autoritäre Lernen der Nachahmung von Strategien sozialer Bewegungen, um Gegenproteste von Loyalist*innen zu mobilisieren. Bereits während der Tahrir-Proteste im Jahr 2011 starteten Mubarak-Anhänger*innen eine Facebook-Seite mit dem Titel »Es tut uns leid, Präsident Mubarak!« und diskreditierten die Demonstrationen als respektloses Verhalten gegenüber dem »Vater der Nation« (Badr 2013). Damals sollten Desinformationskampagnen von »bezahlten elektronischen Komitees « gefälschte Nachrichten und Gerüchte verbreiten, mit dem Ziel, die digitale Öffentlichkeit zu entpolitisieren (ebd.).

Unter diesen eingeschränkten Bedingungen führt der Anstieg der Nutzer*innenzahlen nicht automatisch zu Protesten, wenn die Bürger*innen die Lage als zu hoffnungslos oder zu riskant für die eigene Sicherheit empfinden. 


\section{3 Über die binäre Logik hinaus: Medien und Transformation durch counter-issues verstehen}

Um die Kontroverse zwischen den Polen »Medien zuerst« und »Politik zuerst« aufzulösen sowie Zusammenhänge zwischen Medien und Transformation zu verstehen, plädiert der Artikel für ein soziopolitisch und regional kontextualisiertes Verständnis des Begriffs counter-issues. Wie bereits erklärt, handelt es sich dabei um Themen, die von Gegeneliten wie der Opposition oder Zivilgesellschaft aufgegriffen werden, um die jeweils herrschenden Regimes zu delegitimieren. In den Kontexten der arabischen Umbrüche umfassen die counter-issues politische Themen wie Menschenrechtsverletzungen, Folter und Korruption oder sozioökonomische Themen wie Regimedysfunktion, Ungerechtigkeit oder unverantwortliche Privatisierung. Das zentrale Argument des Beitrags lautet, dass die Transformationsprozesse auf mediale Aufmerksamkeit und Sichtbarkeit von counter-issues zurückzuführen sind und dass weder Medien noch Politik allein die Transformationsprozesse auslösten. Mittels der Kommunikation in hybriden Mediensystemen delegitimierten Akteur*innen öffentlich die Regimes in Ägypten und Tunesien. Trotz der asymmetrischen Machtverhältnisse reagierten diese langsam, so dass die Strategien der Aktivist*innen Erfolg zeigten. Die Medien reformierten die Beziehungen und vermittelten nicht nur Ideen auf lineare Weise. Medientechnologie allein hat keine Relevanz, wenn es keine Akteur*innen des Wandels gibt, deren Diskurse, Handlungen und Zugang zu Medien die auferlegten Beschränkungen überwinden.

\subsection{Interdependenzen zwischen Medien und ihren politischen und gesellschaftlichen Kontexten}

Die Untersuchung der Interdependenzen von Medien und ihren Kontexten in den Bereichen Politik, Kultur und Gesellschaft hilft uns, politische Wandelprozesse besser zu verstehen. Da Medienaufmerksamkeit in einer übersättigten, hybriden Umgebung eine knappe Ressource ist (Tufekci 2013), erhöhen die Aktivist*innen die Sichtbarkeit ihrer Themen durch diverse Protestrepertoires und kommunikative Strategien und verschaffen so ihren counter-issues in einer breiteren Öffentlichkeit Gehör. Soziale Medien komprimieren Zeit und Raum dramatisch, indem die Nutzer*innen vermittelst ihrer wachsenden Cross-media-Nut- 
zung die räumlichen und medialen Grenzen überschreiten (Kaun 2015). Die Sichtbarkeit der counter-issues ging über den Kreis enger Sympathisant*innen hinaus in eine breitere Öffentlichkeit. Gleichzeitig gelang es den Machthabern nicht, diese Sichtbarkeit zu unterdrücken oder ihr entgegenzuwirken. Im Gegenteil hatte die Repression, je nach Thema, eine noch stärkere Mobilisierung zur Folge, wie zum Beispiel bei der Polizeifolter oder sozioökonomischen Arbeiter*innenstreiks.

Die Entwicklung eines Anti-Folter-Aktivismus in Ägypten seit Mitte der 2000er Jahre zeigt, wie sich die Aufdeckung der Polizeibrutalität im bekannten Fall von Khaled Said durch den Einfluss der hybriden Medien intensivierte. Der junge Mann aus Alexandria, der im Juni 2010 von Polizeibeamten zu Tode geprügelt wurde, erschütterte die moralische Grundlage von Mubaraks Herrschaft (Badr 2019). Da sich der tragische Vorfall vor unzähligen Zeugen am helllichten Tag ereignete, sorgte er schon für Aufruhr und Protest in Alexandria, bevor er überhaupt in den Medien bekannt wurde. Ähnlich hat die Erschießung von Demonstrierenden in Tunesien im Dezember 2010 die mediale Sichtbarkeit und die Aufregung über die Ungerechtigkeit in der nicht-politisierten Öffentlichkeit erhöht. Ohne die Thematisierung durch oppositionelle Aktivist*innen wäre es nicht möglich gewesen, die Wut der Massen auf diese Weise zu mobilisieren.

\subsection{Kontextualisierte Zusammenhänge in hybriden Mediensystemen und strukturelle Rahmenbedingungen der politischen Öffentlichkeit}

Die Erforschung der Zusammenhänge von hybriden Medien erklärt die Transformation in Nordafrika, ohne dabei die neuen Medien überzubewerten oder von ihrem Kontext zu isolieren. Das Erforschen der Sichtbarkeit von counter-issues muss digitale und nicht-digitale Medien innerhalb eines hybriden Systems integrieren. Wenn man die Hybridität der Medien übersieht, vernachlässigt man das weitere Umfeld. Die Verortung der Medien in ihre gesellschaftspolitischen und kulturellen Kontexte und die Anerkennung ihres Dynamikpotenzials führt zu kontextualisierten Ergebnissen. So können counter-issues innerhalb gesellschaftlicher Diskurse rekonstruiert werden. Digitale Medien isoliert zu untersuchen führt zu den blinden Flecken, die in der oben ausgeführten »Medien-zuerst«-These erwähnt wurden. 
Zurück zum Fall Khaled Said in Ägypten: Die Analyse der Medieninhalte und Strategien der Aktivist*innen zeigt, dass private Printmedien eine entscheidende Rolle beim Sichtbarmachen von Gerechtigkeitsnarrativen spielten. Obwohl die staatlichen Medien versuchten, das Thema komplett zu ignorieren oder herunterzuspielen, musste das Regime angesichts der Medienaufmerksamkeit und der andauernden Proteste reagieren und die angeklagten Polizeibeamten vor Gericht stellen.

Eine Fokussierung auf die Rolle der Sozialen Medien allein, zum Beispiel die Facebook-Seite »We are all Khaled Said«, würde die hohe Mediensichtbarkeit außerhalb Facebooks, das damals eine noch geringe Nutzer*innenzahl umfasste, nicht erklären. Denn gerade das counterissue »Polizeigewalt« hat eine Vorgeschichte in nicht-digitalen Medien, wurde es doch seit Jahren von zahlreichen zivilgesellschaftlichen Akteur*innen und Netzwerken von Aktivist*innen bearbeitet. Dieser weltbekannte Fall verweist auf das oft übersehene Intermedia-Agenda-Setting: Während sich die Berichterstattung über den Vorfall verzögerte, zirkulierte das grausige Bild des getöteten jungen Mannes zunächst in einem kleinen Kreis von Blogger*innen. Ein ehemaliger oppositioneller Präsidentschaftskandidat veröffentlichte es daraufhin auf seinem Facebook-Profil (Ghonim 2012). Die regelmäßigen »Silent Stands in Black«, Akte des nicht-konfrontativen Widerstands im Sommer 2010, breiteten sich schnell in Kairo aus und zogen zahlreiche Sympathisant*innen an, die sich vorher nicht kannten. In diesen friedlichen Protestaktionen standen junge Demonstrant*innen still vor dem Meer in Alexandria oder am Nil in Kairo, mit dem Rücken der Straße zugewandt, um der Polizeiopfer zu gedenken. Diese Methode sollte eine Konfrontation mit der Polizei meiden und auf Provokationen verzichten (Ghonim 2012).

Die Printmedien konnten das Ereignis also nicht mehr ignorieren: Sobald das Schweigen gebrochen war, erzeugte die mediale Sichtbarkeit des counter-issues eine hohe Aufmerksamkeit, die schließlich sogar eine Thematisierung in den abendlichen Talkshows zur Folge hatte und somit ein noch breiteres Publikum erreichte. So wurde Khaled Said posthum zum Symbol der Tahrir-Revolution, auch wenn er bereits sechs Monate zuvor getötet worden war.

Die strukturellen Rahmenbedingungen der politischen Ökonomie der Medien spielten eine wesentliche Rolle bei der Funktion des Intermedia-Agenda-Setting. Denn der semi-liberalisierte kontrollierte Pluralismus der privaten Massenmedien förderte die Entwicklung eines professionellen Journalismus, der die publikumsrelevanten Themen aufgreifen musste, um seine gesellschaftliche Relevanz sowie seine 
Verkaufszahlen beizubehalten (Badr 2019). Somit trugen sie zur Liberalisierung der Presselandschaft bei und förderten die Medienfreiheit selbst unter semi-autoritären Bedingungen. Dies belegt, wie wichtig die Unterstützung eines unabhängigen professionellen Journalismus ist, und erklärt auch, warum autoritäre Regimes die politische Ökonomie der Medienindustrie - selbst in digitalen Zeiten - zu kontrollieren versuchen.

Weiterhin förderte die damals noch unregulierte digitale Öffentlichkeit das Entstehen kritischer Diskurse. Vor den arabischen Umbrüchen griffen die Mediengesetze zu kurz und waren nicht darauf ausgelegt, oppositionelle Onlinediskurse zu verfolgen. Deshalb strebten Regimes in der Phase der Re-Autokratisierung eine Etablierung neuer restriktiver Medienregulierungsinstanzen und Gesetze an, um digitale Öffentlichkeiten strenger kontrollieren und abweichende Diskurse, zum Beispiel durch Anti-Terror-Gesetze, bestrafen zu können (Badr 2020).

\subsection{Aufstände sind Prozesse, keine plötzlichen »außergewöhnlichen« Momente}

Der Großteil der Literatur über die arabischen Umbrüche deutete Aufstände als einen plötzlichen unvorhersehbaren Moment in der Geschichte (Bayat 2013). Diesen Moment der Massenmobilisierung als eine Überraschung zu beschreiben übersieht jedoch die Vorgeschichte des Aktivismus: Die Akteur*innen hatten langjährige Erfahrung mit Widerstands- und Medienpraktiken unter repressiven Bedingungen. So kamen die arabischen Umbrüche nicht aus dem Nichts, sondern bildeten Höhepunkte eines andauernden Prozesses der Herausforderung von Unrechtsregimen und der Etablierung gerechter Gesellschaften (El-Hamalawy 2019). Der Nährboden war und ist immer noch gegeben: Marginalisierung, sozioökonomische Ungleichheit und prekäre Lebensbedingungen sowie mangelnde Aushandlungsprozesse in der Öffentlichkeit.

Vergleicht man zwei nordafrikanische Staaten - Ägypten und Tunesien -, die 2011 einen temporären Regimekollaps erlebt hatten, so erkennt man sowohl übergreifende Gemeinsamkeiten als auch regionale Unterschiede. Beide Länder standen jahrzehntelang unter der Herrschaft von Autokraten, ohne die klare Perspektive eines möglichen Machtwechsels. Die neoliberale Medienpolitik im Namen der Modernisierung verursachte in diesen Herrschaftsregimes tiefe Legitimitätskrisen, die durch die sozioökonomischen, politischen und menschen- 
rechtlichen Missstände noch verschärft wurden. Zudem sorgte die strukturelle Marginalisierung von Jugendlichen, die immerhin die demographische Mehrheit in den arabischen Gesellschaften ausmachen, für die Aushöhlung ihrer Herrschaftsbasis (Ali und El-Sharnouby 2014; Ismail 2012).

Trotz der scheinbaren Stagnation heute sieht die Perspektive »Revolution als Prozess « (Hamed 2014) die Möglichkeit für eine systembedingt notwendige Transformation und eine erneute Dynamik, da die gesellschaftlichen Konstellationen bis dato nicht nachhaltig stabil und vor allem nicht gerecht sind. Transformation als Prozess bedeutet, dass aufgrund des massiven Destabilisierungspotenzials, welches die sozioökonomische strukturelle Ungleichheit birgt (Weipert-Fenner 2021), neue disruptive Momente aufkommen werden, sobald sich die Gelegenheit dazu ergibt. Eine kontextsensitive Analyse von subversiven und subtilen Mikropraktiken von Medienaktivismus aus Nordafrika liefert empirische Hinweise für diesen langen prozesshaften Charakter der politischen Transformation und dekonstruiert eine schwarz-weiße Sicht der Mainstream-Demokratisierungsforschung. Denn Akteur*innen handeln selbst unter restriktiven Bedingungen in kleineren politischen Gegenöffentlichkeiten, die durch gegenseitiges Vertrauen und Geschlossenheit geprägt sind und somit unter dem Radar der Regimes agieren (Abidin 2021).

Wenn oppositionelle Akteur*innen mit harten repressiven Konsequenzen rechnen, wenden sie subversive Medientaktiken an. So machten sie zum Beispiel von anonymen E-Mail-Verteilern und geheimen Facebook-Gruppen Gebrauch oder operierten aus dem Exil. Vor 2011 nutzten die Aktivist*innen jedoch auch den Duldungsspielraum des Regimes aus. In Ägypten hat die Privatisierung der Medien- und Kulturszene seit Mitte der 1990er Jahre die politischen und medialen Rahmenbedingungen in gewisser Hinsicht gelockert. Literatur und Film öffneten Räume medialer Sichtbarkeit für counter-issues wie Polizeigewalt, Korruption und Ungleichheit für ein breiteres Publikum jenseits direkter politischer Kontrolle. Ersteres wurde zum Beispiel in Alaa alAswanis Roman Der Jakubijān-Bau und Youssef Chahines Film Chaos medial sichtbar. Durch diese kulturellen Diskurse sickerten counterissues in die Öffentlichkeit ein. Die fiktionalen Erzählungen basierten dabei oft auf wahren Geschichten, die zuvor von Aktivist*innen und Blogger*innen enthüllt worden waren.

Ein weiteres Beispiel für Mikroprozesse der Transformation ist das verdeckte Zusammenspiel zwischen Journalismus und der Aktivist*in- 
nenszene. Interviewte Journalist*innen im Rahmen dieses Forschungsprojekts berichten von Überlebens- und Berichterstattungstaktiken in der Nachrichtenredaktion, die es ihnen ermöglichten, über Proteste und counter-issues zu schreiben, indem sie befreundete Aktivist*innen dazu aufforderten, ein bestimmtes Statement in den Sozialen Medien zu veröffentlichen, so dass sie dieses legitim wiedergeben oder zitieren konnten. Im deutlich restriktiveren Tunesien vor 2011 neigten Journalist*innen oppositioneller Zeitungen dazu, die kritische Berichterstattung in kleinerer Schrift in der Nachrichtenrubrik zu verstecken: »Die Leser*innen wussten jedoch genau, wo sie suchen mussten!«, erklärte der ehemalige Chefredakteur einer linken Zeitung. Empirische Hinweise zeigen, wie Mikropraktiken selbst unter stagnierenden Verhältnissen möglich sind. Die Transformation als »Revolution im Prozess « zu sehen bildet deshalb einen geeigneteren Analyserahmen als die binäre Konstruktion von »Autokratien versus Demokratien«.

\subsection{Mehr innerarabische Vergleiche zur regionalen Differenzierung}

Die Länder Nordafrikas unterscheiden sich trotz gewisser sprachlicher, historischer und kultureller Ähnlichkeiten in ihren lokalen Dynamiken teils erheblich. Insgesamt ist die Erforschung von Medien und Kommunikation in der arabischen Region noch wenig vorangeschritten, was durch eine Diversifizierung der Wissensproduktion langfristig behoben werden sollte, um mehr Regionen, die nicht dem Westen zugehören, in den Fokus zu rücken und den dort ansässigen Akteur*innen Gehör zu verschaffen (Chakravartty et al. 2018; Badr et al. 2020; Richter und Kozman 2021). Im Kontext der Transformation bedeutet das, dass wir mehr regional vergleichende Forschung brauchen. Auf den ersten Blick sind Parallelen auf der Makroebene in den Ländern der arabischen Umbrüche nicht zu übersehen: Inkongruenz der politischen Struktur und Kultur, technikaffine Jugend, sozioökonomische Ungleichheit sowie die Zentralisierung der Macht in den Hauptstädten prägen Ägypten und Tunesien gleichermaßen. Doch kontextspezifische Differenzen gestalteten die Verlaufsdynamik der Umbrüche auf andere Art und Weise. Einige wesentliche Unterschiede zwischen beiden nordafrikanischen Ländern ist die Stärke und Vernetzung der Zivilgesellschaft, zum Beispiel von Gewerkschaften, das Bildungsniveau und die Inklusion von Frauen in der Politik. Diese Faktoren begünstigten eine friedlichere - 
wenn auch turbulente - Transformationsphase in Tunesien. Auch prägte dort das stark ausgeprägte postkoloniale Erbe die Medienlandschaft, die in zwei Sprachen (Arabisch für die Massen und Französisch für die Eliten) gespalten war. Die Pakte zwischen den politischen Parteien sowie die Verbindungen zu regionalen und internationalen Akteur*innen definierten die Machtdynamik und die politische Transformation im postrevolutionären Tunesien.

Selbst der Verlauf der Umbruchsdynamik sowohl in Ägypten als auch in Tunesien vor 2011 weist große Unterschiede auf. Der Vergleich beider Staaten zeigt interessante Muster: Zunächst waren die strukturellen sozioökonomischen Ursachen der Arbeiter*innenstreiks von 2008 ähnlich. In beiden Ländern beklagte man prekäre Arbeitsverhältnisse: So protestierten die Arbeiter*innen in Ägypten gegen die Privatisierung, während sie in Tunesien größere Beschäftigungsmöglichkeiten und die Entwicklung der ressourcenreichen Provinzen forderten. In beiden Ländern beeinflusste zudem die geographische Lage die Mediensichtbarkeit und die Reaktionen der Regimes auf diese Proteste. Gegenüber Streiks im tunesischen Gafsa, einer abgelegenen südlichen Provinz, reagierte das Regime mit brutaler Repression und Abschottung. Anders war es hingegen in Ägypten: Dort versuchte die Regierung in der bevölkerungsreichen Nildelta-Stadt Mahalla, einem Zentrum der Textilindustrie, das geographisch viel näher an der Hauptstadt liegt, Verhandlungen aufzunehmen. Die unterschiedlichen Reaktionen der Regimes haben auch medienrelevante Ursachen: Die streng zensierte Medienlandschaft Tunesiens thematisierte die Proteste kaum, weshalb diese nur in oppositionellen Medien mit begrenzter Reichweite Verbreitung fanden. Die ägyptische Regierung war andererseits gezwungen zu reagieren, da die Verbindung zwischen den Arbeiter*innen und der städtischen Jugend zu einer kurzlebigen klassenübergreifenden Solidarität führte. Insofern führte das Zusammenspiel von alten und neuen Medien sowie klassischen und innovativen Protesttechniken zu unterschiedlichen Reaktionen der Regimes, die durch die Medienaufmerksamkeit auf die counter-issues noch verstärkt wurden.

\section{$4 \quad$ Fazit und Ausblick}

Die Erforschung des Zusammenhangs von Transformation und Medien ist im Fach der Kommunikationswissenschaft relevanter denn je. Aus der arabischen Region kann noch vieles gelernt werden. Dazu gehört 
erstens, dass es das Konzept der counter-issues ermöglicht, kontextsensible Medienanalysen zu Wandelprozessen durchzuführen und auf eine technikzentrierte Interpretation zu verzichten, die sich zu sehr auf digitale Technologien beschränkt und dabei die soziokulturellen und politischen Hintergründe vernachlässigt. Die Berücksichtigung der Interaktionen verschiedener Medienformate vor dem Hintergrund von Dynamik und Hybridität überwindet die Trennung zwischen Onlineund Offlinemedien. Somit entwickeln sich Themen in hybriden Medienumgebungen, indem sie von Akteur*innen trotz autoritärer Strukturen lanciert werden.

Selbst in eingeschränkten Kontexten spielen die Medien eine Rolle bei der öffentlichen Thematisierung von Missständen. Im Zuge eines jeden erfolgreichen Protests neigt die akademische Debatte dazu, schnell zur These des Liberalisierungseffektes der Medien zu greifen, anstatt eine kontextualisierte und regional differenzierte Debatte anzuregen. Es geht darum, dass Medien und Transformation als Prozesse zu betrachten sind. Dabei ist es hilfreich, nicht nur die Akteur*innen zu betrachten, sondern auch die Reaktionen der Machthaber einzuschließen, um die dynamischen Wechselwirkungen und Lernprozesse der beteiligten Parteien zu erfassen. Vor diesem Hintergrund sieht sich die arabische Region unverändert zahlreichen Herausforderungen gegenüber, denn die gegenwärtigen Ungerechtigkeiten sind langfristig nicht tragbar. So besteht weiterhin das Potenzial für erneute Protestwellen und Transformationsprozesse, solange die strukturellen Ursachen der Unzufriedenheit nicht behoben werden. Punktuelle Proteste - trotz hoher politischer Kosten der Repression - belegen diese Instabilität der sozioökonomischen Konstellationen.

Weiterhin fordert dieser Beitrag dazu auf, die regional vergleichende Medien- und Kommunikationswissenschaftsforschung voranzutreiben, anstatt lediglich einzelne Fallstudien zu analysieren. Bislang ist das Potenzial der innerarabischen kommunikationswissenschaftlichen Forschung, anders als etwa in der Politikwissenschaft, noch nicht ausgeschöpft. Zwar besteht in der westlichen akademischen Welt ein größeres Interesse daran, den globalen Norden zu erforschen. Doch um die globale Dimension der Zusammenhänge zwischen Digitalisierung und Öffentlichkeiten zu verstehen, brauchen wir mehr Forschung direkt aus und über nicht-westliche Kontexte. Die regionale Differenzierung deckt Muster auf, die unseren epistemischen Horizont erweitern.

Schließlich sollten wir aus der Forschungswelle zu Medien und den arabischen Umbrüchen lernen, die Transformation als Prozess und 
nicht als Momentaufnahme zu sehen. Selbst unter eingeschränkten autoritären Systembedingungen entstehen subversive Taktiken des Widerstands, die über die kurzlebigen disruptiven Umbruchsmomente hinaus forschungsrelevant sind. Trotz der gegenwärtigen Re-Autokratisierung und Verzweiflung in der Region wissen wir, dass es solche Momente des Aufbruchs gab und wieder geben wird. Wir können Ereignisse wie die des Jahres 2011 nur verstehen, wenn wir auch die Erforschung von Kommunikation in autoritären Kontexten als relevant betrachten (Töpfl 2020). Zwar schränkt die restriktive politische Ökonomie der Medien eine transparente Öffentlichkeit ein, aber die kreative Energie der handelnden Akteur*innen und ihre Widerstandsbemühungen, subversiv kulturelle und soziale Bereiche zu beeinflussen, bleiben ebenso beständig und bemerkenswert. Eine solche kontextualisierende Perspektive überwindet eine binäre Sichtweise auf die Rolle der Medien in Wandelprozessen und verhilft uns zu einem differenzierten Verständnis von Medien in Zeiten der digitalen Transformation.

\section{Literatur}

Abdulla, Rasha A. 2011. The Revolution Will Be Tweeted. The Cairo Review of Global Affairs. https://www.thecairoreview.com/essays/the-revolution-will-betweeted/. Zugegriffen: 20. Februar 2021.

Abidin, Crystal. 2021. From »Networked Publics« to »Refracted Publics«: A Companion Framework for Researching »Below the Radar« Studies. Social Media + Society. DOI: $10.1177 / 2056305120984458$.

Ali, Amro, und Dina El-Sharnouby. 2014. Distorting digital citizenship: Khaled Said, Facebook, and Egypt's streets. In: Wired Citizenship: Youth Learning and Activism in the Middle East, herausgegeben von Linda Herrera, 89-101. New York: Routledge.

Aswani, Alaa A. 2007. Der Jakubijān-Bau. Übersetzt von Hartmut Fähndrich. Basel: Lenos-Verlag.

Ayish, Muhammad I. 2008. The New Arab Public Sphere. Medien und politische Kommunikation - Naher Osten und islamische Welt. Band 15, herausgegeben von Kai Hafez, und Carola Richter. Berlin: Frank \& Timme.

Badr, Hanan. 2013. Battleground Facebook: Contestation Mechanisms in the Social Media in the Framing of the Egypt's Revolution 2011. In: Social Media Go to War: Rage, Rebellion and Revolution in the Age of Twitter, herausgegeben von Ralph D. Berenger, 399-422. Spokane: Marquette Books.

Badr, Hanan. 2015. Limitations of the Social Media Euphoria in Communication Studies. Égypte/Monde arabe 12. DOI: 10.4000/ema.3451.

Badr, Hanan. 2019. Before the »Arab Spring«: How challengers pushed counter-is- 
sues in Egypt's hybrid media system. Media, War and Conflict. DOI: $10.1177 / 1750635219894611$.

Badr, Hanan. 2020. From Disruptive Power to Trapped Endurance: Egypt's Journalistic Agency after the Tahrir Revolution. In: Critical Incidents in Journalism: Pivotal Moments Reshaping Journalism Around the World, herausgegeben von Edson C. Tandoc Jr., Joy Jenkins, Ryan J. Thomas und Oscar Westlund. Milton Park: Routledge.

Badr, Hanan, Markus Behmer, Susanne Fengler, Anke Fiedler, Anne Grüne, Kai Hafez, Oliver Hahn, Kefa Hamidi, Thomas Hanitzsch, Christine Horz, Beate Illg, Anna Litvinenko, Martin Löffelholz, Melanie Radue, Carola Richter, Barbara Thomaß, und Florian Töpfl. 2020. Kosmopolitische Kommunikationswissenschaft: Plädoyer für eine »tiefe Internationalisierung « des Fachs in Deutschland - Ein wissenschaftspolitisches Positionspapier. Publizistik 65: 295-303. DOI: 10.1007/s11616-020-00576-6.

Bayat, Asef. 2013. The Arab Spring and its Surprises. Development and Change 44 (3): 587-601. DOI: 10.1111/dech.12030.

Beck, Martin. 2015. The End of Regional Middle Eastern Exceptionalism? The Arab League and the Gulf Cooperation Council after the Arab Uprisings. Democracy and Security 11 (2): 190-207. DOI: 10.1080/17419166.2015.1037390.

Bellin, Eva. 2012. Reconsidering the Robustness of Authoritarianism in the Middle East: Lessons from the Arab Spring. Comparative Politics 44 (2): 127-149. DOI: $10.5129 / 001041512798838021$.

Chadwick, Andrew. 2017. The Hybrid Media System: Politics and Power. 2. Auflage. New York: Oxford University Press.

Chakravartty, Paula, Rachel Kuo, Victoria Grubbs, und Charlton McIlwain. 2018. \#CommunicationSoWhite. Journal of Communication 68 (2): 254-266. DOI: 10.1093/joc/jqy003.

Chaos. Ägypten und Frankreich 2007. Regie: Youssef Chahines und Khaled Youssef. DVD 3B Productions und MISR International Films.

Diamond, Larry. 2010. Liberation Technology. Journal of Democracy 21 (3): 69-83.

El-Hamalawy, Hossam. 2019. Revolution is a process, and it's far from over. The New Arab vom 27. Februar, https://english.alaraby.co.uk/english/comment/2019/2/27/revolution-is-a-process-and-its-far-from-over. Zugegriffen: 20. Februar 2021.

El-Issawi, Fatima. 2021. Alternative Public Spaces in Hybrid Media Environments: Dissent in High Unvertainty. Journalism \& Mass Communication Quarterly. DOI: 10.1177/1077699021998381.

El-Nawawy, Mohammed, und Sahar Khamis. 2013. Egyptian Revolution 2.0: Political Blogging, Civic Engagement, and Citizen Journalism. New York: Palgrave Macmillan.

Elswah, Mona, und Philip N. Howard. 2020. The Challenges of Monitoring Social Media in the Arab World: The Case of the 2019 Tunisian Elections. Oxford Internet Institute, Oxford University: Project on Computational Propaganda. https:// comprop.oii.ox.ac.uk/wp-content/uploads/sites/93/2020/03/Tunisia-memoEnglish.pdf. Zugegriffen: 20. Februar 2021. 
Ghonim, Wael. 2012. Revolution 2.0. Cairo: Shorouk.

Hafez, Kai. 2014. Radikale Polarisierung im Arabischen Frühling: Aufstieg und Niedergang der Medienfreiheit in Ägypten. Sicherheit und Frieden 32 (4): 254259.

Hamed, Adham. 2014. Revolution as a Process: The Case of the Egyptian Uprising. Wien: Wiener Verlag für Sozialforschung.

Hamzawy, Amr. 2017. Egypt after the 2013 military coup: Law-making in service of the new authoritarianism. Philosophy \& Social Criticism 43 (4/5): 392-405. DOI: $10.1177 / 0191453717695367$.

Harders, Cilja. 2018. Understanding Egypt's Postrevolutionary dynamics »from below«. In: Ways of Knowing Muslim Cultures and Societies: Studies in Honour of Gudrun Krämer, herausgegeben von Bettina Gräf, Birgit Krawietz und Schirin Amir-Moazimi, 411-424. Leiden: Brill Academic Publishers.

Harik, Iliya. 2006. Democracy, »Arab Exceptionalism«, and Social Science. The Middle East Journal 60 (4): 664-684. DOI: 10.3751/60.4.12.

Howard, Philip N., und Muzammil M. Hussain. 2013. Democracy's Fourth Wave? Digital Media and the Arab Spring. New York: Oxford University Press.

Ismail, Salwa. 2012. The Egyptian revolution against the police. Social Research 79 (2): 435-462.

Kaun, Anne. 2015. Regimes of time: Media practices of the dispossessed. Time \& Society 24 (2): 221-243. DOI: 10.1177/0961463X15577276.

Khamis, Sahar, und Katherine Vaughn. 2011. We Are All Khaled Said: The Potentials and Limitations of Cyberactivism in Triggering Public Mobilization and Promoting Political Change. Journal of Arab \& Muslim Media Research 4 (2/3): 145-163. DOI: 10.1386/jammr.4.2-3.145_1.

Kneuer, Marianne, und Thomas Demmelhuber. 2016. Gravity centres of authoritarian rule: a conceptual approach. Democratization 23 (5): 775-796. DOI: 10.1080/13510347.2015.1018898.

Lynch, Marc. 2014. The Arab Uprising Explained: New Contentious Politics in the Middle East. New York: Columbia University Press.

Maoz, Zeez, und Bruce Russett. 1993. Normative and Structural Causes of Democratic Peace, 1946-1986. American Political Science Review 87 (3): 624-638. DOI: $10.2307 / 2938740$.

Mathes, Rainer, und Barbara Pfetsch. 1991. The Role of the Alternative Press in the Agenda-Building Process: Spill-over Effects and Media Opinion Leadership. European Journal of Communication 6 (1): 33-62. DOI: 10.1177/026732319100 6001003.

Merkel, Wolfgang. 2010. Systemtransformation: Eine Einführung in die Theorie und Empirie der Transformationsforschung. Wiesbaden: Springer VS.

O'Donnell, Guillermo, und Philippe C. Schmitter. 2013. Tentative Conclusions about Uncertain Democracies. Baltimore: The Johns Hopkins University Press.

Pfetsch, Barbara. 2018. Dissonant and Disconnected Public Spheres as Challenge for Political Communication Research. Javnost - The Public 25 (1/2): 59-65. DOI: 10.1080/13183222.2018.1423942.

Pfetsch, Barbara, Daniel Maier, Peter Miltner, und Annie Waldherr. 2016. Chal- 
lenger Networks of Food Policy on the Internet: A Comparative Study of Structures and Coalitions in Germany, the UK, the US, and Switzerland. International Journal of E-Politics 7 (1): 16-36. DOI: 10.4018/IJEP.2016010102.

Ragnedda, Massimo, und Glenn W. Muschert. 2016. Theorizing Digital Divides and Inequalities. In: Social Inequalities, Media, and Communication: Theory and Roots, herausgegeben von Jan Servaes und Toks Oyedemi, 23-35. Lanham: Lexington Books.

Raoof, Ramy. 2017. State surveillance and protest: »They try to make people think twice before taking to the streets«. Egyptian Initiative for Personal Rights. https://eipr.org/en/blog/ramy-raoof/2017/10/state-surveillance-and-protest\%E2\%80\%9Cthey-try-make-people-think-twice-taking. Zugegriffen: 20 . Februar 2021.

Richter, Carola, und Hanan Badr. 2018. Communication Studies in Transformation: Self-Reflections on an Evolving Discipline in Times of Change. In: Academia in Transformation: Scholars Facing the Arab Uprisings, herausgegeben von Florian Kohstall, Carola Richter, Sarhan Dhouib und Fatima Kastner, 143-160. Baden-Baden: Nomos.

Richter, Carola, und Claudia Kozman. 2021. Arab Media Systems. Cambridge: Open Book Publishers.

Rinke, Eike M., und Maria Röder. 2011. Media Ecologies, Communication Culture, and Temporal-spatial Unfolding: Three Components in a Communication Model of the Egyptian Regime Change. International Journal of Communication 5: 1273-1285.

Rosato, Sebastian. 2003. The Flawed Logic of Democratic Peace Theory. American Political Science Review 97 (4): 585-602.

Said, Edward. 2003 [1978]. Orientalism. London: Penguin Books.

Salem, Fadi. 2017. The 7th Arab Social Media Report: Social Media and the Internet of Things - Towards Data-Driven Policymaking in the Arab World: Potential, Limits and Concerns. https://www.arabsocialmediareport.com/News/description. aspx?NewsID=24\&PriMenuID=15\&mnu=Pri. Zugegriffen: 11. März 2021.

Strippel, Christian. 2020. Strukturwandel der Öffentlichkeit aus der Perspektive einer Theorie der Spätmoderne. In: Digitaler Strukturwandel der Öffentlichkeit: Historische Verortung, Modelle und Konsequenzen, herausgegeben von Mark Eisenegger, Marlis Prinzing, Patrik Ettinger und Roger Blum, 213-238. Wiesbaden: Springer VS.

Teti, Andrea, Pamela Abbott, Valeria Talbot, und Paolo Maggiolini. 2020. Democratisation against Democracy: How EU Foreign Policy Fails the Middle East. Cham: Palgrave Macmillan.

Töpfl, Florian. 2020. Comparing Authoritarian Publics: The Benefits and Risks of Three Types of Publics for Autocrats. Communication Theory 30 (2): 105-125. DOI: $10.1093 / \mathrm{ct} / \mathrm{qtz} 015$.

Trappel, Josef. 2019. Inequality, (new) media and communications. In: Digital media inequalities: Policies against divides, distrust and discrimination, herausgegeben von Josef Trappel, 9-30. Göteborg: Nordicom.

Tufekci, Zeynep. 2013. »Not This One«: Social Movements, the Attention Economy, 
and Microcelebrity Networked Activism. American Behavioral Scientist 57 (7): 848-870. DOI: 10.1177/0002764213479369.

Weipert-Fenner, Irene. 2021. Nichts ist vorbei! Frankfurter Rundschau vom 11. Januar, https://www.fr.de/meinung/nichts-ist-vorbei-90164970.html. Zugegriffen: 20. Februar 2021.

Wolfsfeld, Gadi, Elad Segev, und Tamir Sheafer. 2013. Social Media and the Arab Spring: Politics Comes First. International Journal of Press/Politics 18 (2): 115137. DOI: $10.1177 / 1940161212471716$.

\section{Open Access}

Dieser Beitrag erscheint unter der Creative-Commons-Lizenz CC BY-ND 3.0 DE: https://creativecommons.org/licenses/by-nd/3.0/de/. 